a chemical transformation with the formation of fluoride has resulted from the continued action of small quantities of sodium fluoride added to the feed water. When once thoroughly cleaned to the metal new scale does not form in such instances.

Live steam is so frequently used now in our large manufacturing establishments for cooking, boiling, etc., and so much condensed steam is used to furnish distilled water, especially in artificial ice manufacture, that a method of treating boiler water in such a way as to furnish pure untainted steam is exceedingly desirable. Since sodium fluoride completes its work without rendering the water alkaline the steam from water thus treated has proved most excellent for such purposes.

It is beyond the province of this article to enter more fully into these details.

\title{
IMPROVED APPARATUS FOR THE VOLUMETRIC DETER- MINATION OF CARBON DIOXIDE AND OTHER GASES.
}

BY CHARLES A CATIIN

OR the determination of small quantities of carbon dioxide
in readily decomposed carbonates, the process and apparatus devised by $\mathrm{Dr}$. Scheibler presents a most convenient and rapid method; but the inability to thus measure large quantities of gas has restricted its employment for the most part to determination of carbonate in bone-char. To extend the field of volumetric carbon dioxide determination I have devised the following described apparatus, retaining, as far as possible, the essential features of the Scheibler.

Referring to the cut: A, C, and D are essentially the same as in the Scheibler apparatus, A, being the decomposing bottle in which the portion of the sample to be operated upon, is placed, with its enclosed tube for the decomposing acid or other solution, with the further addition however, of a thermometer, inserted through the rubber stopper-a most important feature when the decomposition results in wide variations of temperature; $\mathrm{C}$, the bottle containing the rubber gas balloon connected

\footnotetext{
1 Described at June, Ioy3. meeting of the Rhode Island Section of the Anerican Chemical society.
} 
with $\mathrm{A}$, and $\mathrm{D}$ the water reservoir with compressing bulb $\mathrm{E}$, exactly as in the Scheibler device. In the Scheibler apparatus however, the volume of the gas evolved is directly determined by the displacement of water in a graduated tube, the capacity of which is, and must be, quite limited.

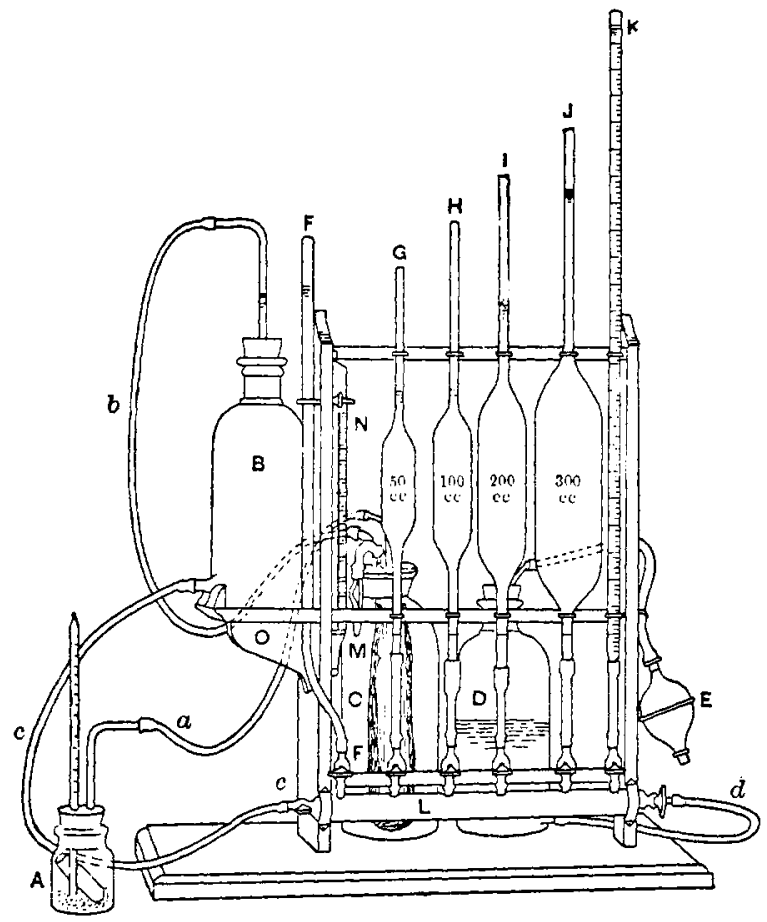

In my apparatus I have replaced this graduated tube by the bottle $\mathrm{B}$, connected by the tube $b$ with the space around the rubber bag in the bottle $C$, and by the tube $c$ from its bottom, through the stopcock, with the tube $\mathrm{L}$, which tube $\mathrm{L}$ connects, through another stopcock at its opposite end, with the reservoir $\mathrm{D}$, by means of the tube $d$. This tube $L$, which may be constructed of brass if more convenient, is fixed horizontally upon the standards, as shown in the cut, and has affixed along its upper portion, a series of stopcocks, one of which connects with the tube $F$, and each of the others individually, with the pi- 
pettes $\mathrm{G}, \mathrm{H}, \mathrm{I}$, and $\mathrm{J},{ }^{*}$ and the burette $\mathrm{K}$. These pipettes may be respectively (using a fifty $\mathrm{cc}$. burette for $\mathrm{K}$ ) 50, 100,200 , and $400 \mathrm{cc}$. capacity, graduated as to contents, between marks on the stem and delivery tube, in each case. The tube $F$ serves as an equalizer of pressure, as hereinafter described. N shows a common tube thermometer attached to the standard for convenient reference.

Before using the apparatus, the reservoir $\mathrm{D}$ is supplied with water, and by means of the compressing bulb $\mathrm{E}$, the burette and pipettes are filled to their zero marks, and their respective stopcocks closed, the stopcocks connecting $c$ and $\mathrm{F}$ being closed meanwhile. The stopcock connecting $c$ is now opened, and the bottle $\mathrm{B}$ filled a little above a zero mark upon the tube $b$, which for a portion of the way is of glass; this glass tube being so inserted in the rubber stopper that the bottle may be entirely filled with water without air space. During the filling of $B$, the stopcock M should be opened, connecting as it does with the air space surrounding the gas bag within the bottle $C$, while the generating bottle $A$ should be disconnected and the gas bag collapsed. When $B$ is filled, the stopcock connecting it with $I_{\text {, }}$ is closed, and that at $\mathrm{F}$ opened, and this tube filled in like manner to a point higher than the zero mark of $B$. when the stopcock connecting $d$ is closed, and that at $c$ opened again. The generating bottle $\mathrm{A}$ with its charge of sample and acid in the tube, is now connected, and through the stopcock connecting $d$ the level of the water in $B$ adjusted to the zero mark, that in F subsiding with it. When the adjustment is thus effected, the stopcock connecting $F$, and that at $M$ are closed, while that connecting with the reservoir through $d$, is opened, and thus free course given for the water between $B$ and $D$. The bottle $B$ being elevated upon the shelf $O$, there is of course a reduction of pressure within the apparatus, causing a subsidence of the water leve1; which after a few moments should cease, showing that the apparatus is tight in every joint. If, however, this is not the case, and the level continues to subside, there is a leak, which must be corrected and a readjustment of pressure effected before the operation is continued.

* By an error of the engraver this pipette is marked $300 \mathrm{cc}$. It should be $400 \mathrm{cc},-\mathrm{E}, \mathrm{D}$. 
When all is in readiness, the decomposition of the carbonate is effected in A by bringing the acid and carbonate together in the usual way, the temperature having been noted at the outset. The evolution of the gas, distending the rubber gas bag, expels a portion of the air from $C$, which forces the water from $B$ into the reservoir $D$, in volume equivalent to the gas evolved at the pressure and temperature prevailing, as is readily understood. When complete decomposition is effected, and the temperature in A returns to the temperature of the room, by means of the bulb $E$, pressure is exerted upon the reservoir $D$, and the stopcock connecting $F$ being opened, the levels in $B$ and $F$ are brought together through the stopcock connecting $d$.

When this is accomplished, the stopcocks at $F$ and $d$ are closed. It is seen that the displacement of the water in $\mathrm{B} \mathrm{ob}$ tained in this manner is exactly that of the volume of the gas evolved from the carbonate at the existing temperature and atmospheric pressure. With all stopcocks closed, excepting that connecting $c$, and that at $\mathrm{M}$ which should now be opened, the bottle $B$ is removed from its shelf to the table upon which the apparatus stands, and the amount of the displacement measured by running in the contents of such of the pipettes, and portion of the contents of the burette, as may be required to restore the water level in $B$ to the starting point, the amount of water thus employed of course being that measure.

From the volume of gas obtained, the percentage by weight of the sample taken is calculated in the usual way, either by the formula for correction of volume for temperature and pressure, adding of course to the volume thus obtained a correction for carbon dioxide dissolved in the decomposing liquid, or through the use of the tables given originally by Dietrich, (Ztschr. anal. Chem., 4, I4I). It is to be noted, however, that these tables are not strictly correct, the weight of the cubic centimeter of carbon dioxide at $0^{\circ} \mathrm{C}$. $760 \mathrm{mgms}$. being considerably at variance with that at present given by the best authorities, and some slight errors, in the calculation apparently, are also to be observed. In ordinary work, however; these are not important. But in the use of the table given for solubility of the gas in the decomposing acid, one must exercise no little caution. In fact 
for this correction, it is better to establish for one's self just what it should be for each material operated upon, by check gravimetric determinations; for while within limits this may be taken as a constant factor, yet it is more or less affected by the character of the salts present. And further, this table was not carried far enough to cover the large volumes of gas evolved from the charges that my apparatus enables one to employ.

With this apparatus it is possible to make a determination of carbon dioxide in from ten to fifteen minutes, and that with extreme accuracy. Indeed with the large charges one may employ, and with careful weighings, repeated results obtained from the same sample, will never vary more than one-tenth per cent. and scarcely more in most instances than two or three one-hundredths per cent.

\section{ARE PENTOSES FORMED BY THE ASSIMILATION PROCESS ?}

DR, G. De Chalmot, Agricultural Departaent, RichmoNd, Va.

$\mathrm{N}$ recent years the chemistry of the sugars having been revoand others, the study of the assimilation process, by which the carbohydrates in the plants are originally formed, has also received a new interest.

The theory of Bayer, according to which the first product of the assimilation is formaldehyde, has recently received a very strong support by the results which Bokorny obtained."

Fischer observed some years ago that glyceric aldehyde was probably an intermediate product in the assimilation process, since it most readily unites with itself, yielding hexoses, when placed under proper circumstances."

Pentosans, substances which yield pentoses by hydrolysis, are most widely distributed in plants and form often a large part of their dry matter. ${ }^{4}$ The pentoses, xylose, and arabinose, belong to the laevo series (of Fischer) in contrast with the more

\footnotetext{
1 Read before the World's Congress of Chemists, August 23, 1893 .

2 Ber. d. bot. Ges., 9, 4, 103.

8 Ber. d. chem. Ges., 23, 2233.

4 Am. Chem, $J, 15,21$, note.
} 